\title{
Current Status of Biportal Endoscopic Decompression for Lumbar Foraminal Stenosis: Endoscopic Partial Facetectomy and Outcome Factors
}

\author{
Hee-Seok Yang ${ }^{1}$, Nam Lee ${ }^{2}$, Jeong-Yoon Park ${ }^{3}$ \\ ${ }^{1}$ Department of Neurosurgery, Seoul Barunsesang Hospital, Seoul, Republic of Korea \\ ${ }^{2}$ Department of Neurosurgery, Yonseicheok Hospital, Busan, Republic of Korea \\ ${ }^{3}$ Department of Neurosurgery, Gangnam Severance Hospital, Yonsei University College of Medicine, Seoul, Republic of Korea
}

Corresponding Author:

Jeong-Yoon Park, MD, $\mathrm{PhD}$

Department of Neurosurgery, Gangnam

Severance Hospital, Yonsei University

College of Medicine, 211, Eonju-ro,

Gangnam-gu, Seoul 06273, Republic

of Korea

Tel: +82-2-2019-3390

Fax: +82-2-3461-9229

Email: spinepjy@yuhs.ac
Degenerative lumbar foraminal stenosis is relatively common condition in which the circumferential narrowing of the space available for the nerve root leads to back pain and radicular symptoms. The preferred surgical treatment to relieve the compression of the nerve root has not been established yet. Recently, several reports have shown good clinical outcomes in patients who underwent biportal endoscopic decompression for the treatment of degenerative lumbar foraminal stenosis. The floating-type biportal endoscopic technique could be used with various surgical instruments without docking in the narrowed foramen, unlike the full-endoscopic technique. Multiple sites can be accessed with more freedom in the approaching angle through triangulation and portal switching. We reviewed articles to understand putative outcome factors and discuss the appropriate indications for biportal endoscopic foraminal decompression. Lumbar lordosis, degenerative lumbar scoliosis, height of the posterior intervertebral disc and level of procedure were all related to clinical outcomes. The best indications and contraindications to the endoscopic foraminal decompression still depends on the surgeon's skill level and evolving experience. However, we could suggest that biportal endoscopic spinal surgery is supposed to be an alternative treatment for foraminal decompression presenving motion and stability, and decreasing the need for fusion surgery in various lumbar degenerative disease.

Key Words: Lumbar foraminal stenosis, Biportal endoscopic spinal surgery, Poor outcome
Received: February 11, 2021

Revised: February 22, 2021

Accepted: March 05, 2021 intervertebral disc eventually results in instability of the spinal segment. The ensuing hypermobility is associated with thickening of soft tissues in the foramen, followed by hypertrophy of the facet joints, particularly at the superior articular process (SAP) ${ }^{27)}$. Radicular symptoms gradually progress insidiously to become painful irritations that are increasingly bothersome, and, eventually debilitating.

Several different surgical techniques for degenerative lumbar foraminal stenosis have been used, including foraminotomy, facetectomy, partial pediculectomy, fusion, and distraction instrumentation. Spinal fusion has been the standard treatment for patients with degenerative lumbar foraminal stenosis associated with preoperative instability or deformity. However, facetpreserving decompression is a more desirable primary treatment for patients with degenerative lumbar foraminal stenosis in the absence of gross instability. With the advancements in minimally invasive surgery, many surgeons have reported good results with facet-preserving decompression and endoscopic partial 
facetectomy in the treatment of degenerative lumbar foraminal stenosis ${ }^{6,13,17)}$. Several reports have recently shown good clinical outcomes in patients who underwent endoscopic decompression of degenerative lumbar foraminal stenosis ${ }^{2,34)}$.

\section{History}

Conventional surgical methods for the treatment of lumbar foraminal or far lateral stenosis can be categorized as total facectectomy with or without fusion and facet-preserving foraminotomy. Total facetectomy offers sufficient decompression through the nerve root course. However, this often leads to segmental instability and back pain ${ }^{15)}$. A facet-preserving microscopic decompression technique using a paraspinal approach was reported by Reulen et al. ${ }^{28)}$ in 1987 and Wiltse and Spencer ${ }^{30)}$ in 1988; other surgeons have since modified this technique. This technique enables direct access to a foraminal or far lateral lesion with minimal violation of the facet joint and minimal postoperative back pain ${ }^{6}$. The success rates reported for open paraspinal foraminotomy are as high as $72 \%$ to $83 \%{ }^{2,9)}$ and this technique has been considered the gold standard for the surgical treatment of lumbar foraminal or far lateral stenosis. However, some patients experience postoperative leg pain or dysesthesia, which are the primary causes of unfavorable outcomes ${ }^{2,9)}$. Moreover, a limited field of view in the paraspinal approach may lead to incomplete decompression. To overcome the limitations of previous surgical techniques, many surgeons have developed various minimally invasive techniques.

Hijikata and Kambin separately introduced percutaneous nucleotomy and Kambin further described the safe triangular zone (Kambin's triangular zone) for docking and working on the transforaminal region ${ }^{16)}$. Various techniques exploring Kambin's triangular zone with uniportal full-endoscopic spine surgery were described, which can be largely summarized as the inside-out, and outside-in with foraminoplasty techniques ${ }^{19)}$. With the development of full-endoscopic techniques, biportal endoscopic decompression techniques using two portals were introduced. Recently, several reports have shown good clinical outcomes in biportal endoscopic decompression and partial facetectomy for degenerative lumbar foraminal stenosis, ${ }^{3,820)}$, effectiveness of endos-

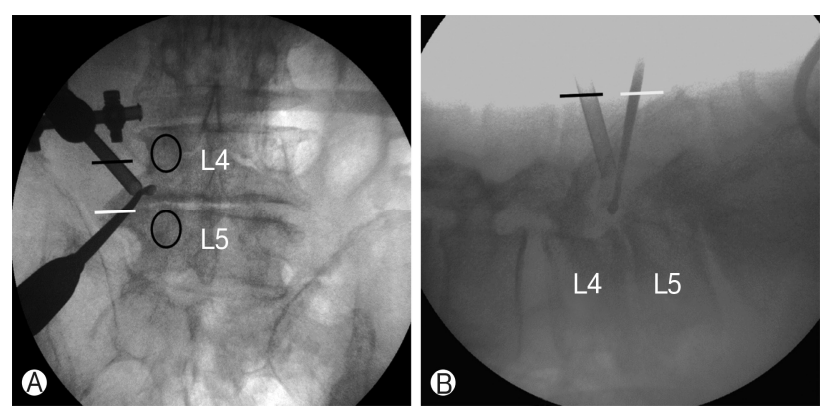

Figure 1. (A): C-arm fluoroscopic image A-P view. Black line: Endoscopic portal incision. White line: instrumental portal incision. Black circle: pedicles. (B): C-arm fluoroscopic image lateral view. copic surgery for foraminal stenosis was proven. The minimal damage to the paraspinal muscles, coupled with short recovery times, reduced blood loss, and good clinical results are the major endoscopic spine surgery advantages for endoscopic decompression $^{2,35}$. During foraminal decompression and partial facetectomy, advantages of the biportal endoscopic surgery over the uniportal full-endoscopic spine surgery are in the visualiza- tion of the intracanalicular region of the lumbar spine. It affords a wider view with more degrees of freedom to manipulate the instruments, whereas the uniportal endoscope restricts the motion of the instruments and obscures visualization, especially in difficult-to-see areas around exiting nerve roots and lateral recesses ${ }^{3,20)}$.

\section{Surgical Procedures}

\section{1) Patient Position, Anesthesia and Skin Incision}

Instruments including standard spine instruments, $0^{\circ}$ and $30^{\circ}$ $4 \mathrm{~mm}$ diameter endoscopes, radiofrequency probe, and endoscopic drills were used. Endoscopes with different viewing angles were used alternately, depending on the operative field. The procedures were operated under epidural or general anesthesia, and the patients were positioned prone, with the abdomen free. Level confirmation was conducted under intraoperative fluoroscopy. Under anteroposterior C-arm fluoroscopic imaging parallel to the upper endplate of the proximal vertebral body at the operative level, the upper and lower pedicles and their transverse process were marked. One-centimeter incisions were made 2 $\mathrm{cm}$ lateral to the lateral margin of the pedicle line on the mid-line of each of the two transverse processes (Figure 1).

\section{2) Insertion of the Endoscope and Continuous Saline Irrigation}

In order to perform biportal endoscopic spine surgery, two portals must be created. Spinal needles were temporarily introduced through the skin incision to confirm triangulation of the viewing portal and working portal under $\mathrm{C}$-arm fluoroscope. Initially, a 0 degree scope was inserted through viewing portal, and a saline irrigation pump was connected to the endoscope, set to a pressure of $25-30 \mathrm{mmHg}$ during the procedure, and controlled within this range depending on the condition of the sur- gical view. Keeping the sheath for the working portal is helpful to maintain stable continuous saline flow. Soft tissues was gently detached from the lateral edge of the isthmus of the lamina, facet joint and transverse process of the targeted intervertebral foramen with a small periosteal elevator or radiofrequency probe. After exposing the lateral edge of the isthmus, facet joint, and transverse process, soft-tissue remnants and bleeding were effec- tively managed by a pituitary rongeur and radiofrequency probes (Figure 2). Because the radicular artery located around the facet joint is easily damaged during this process, which can cause massive bleeding, it could be advantageous to coagulate beforehand carefully (Figure 3 ). 


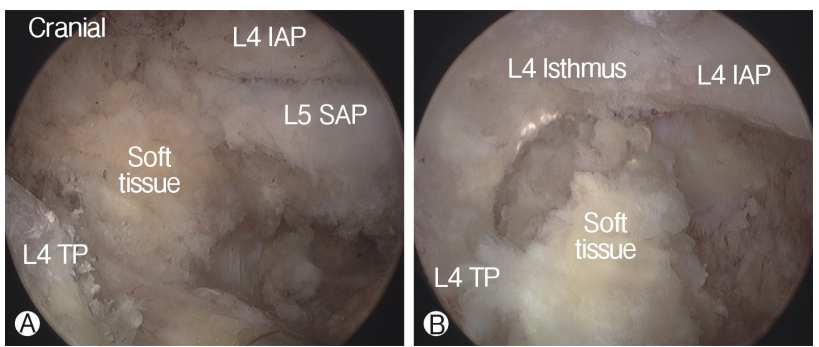

Figure 2. (A): Left-side approach endoscopic foraminal view. After making initial working space, physician can identify the foraminal anatomy. (B): After partial resection of SAP tip.
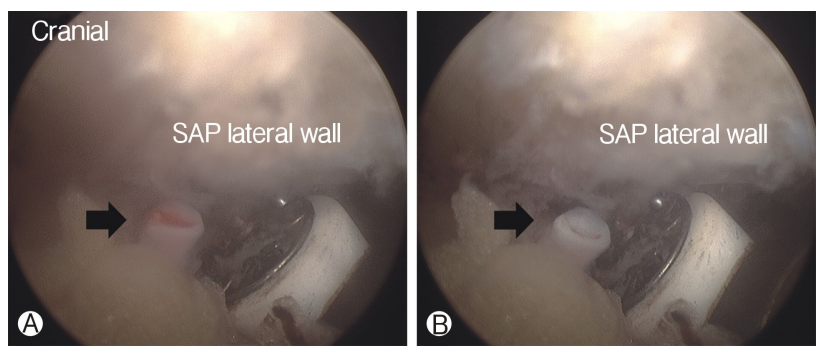

Figure 3. Left-side approach. The radicular artery located laterally of superior articular process (SAP). Black arrow shows radicular artery before coagulation (A) and after coagulated state (B) by RF probe.
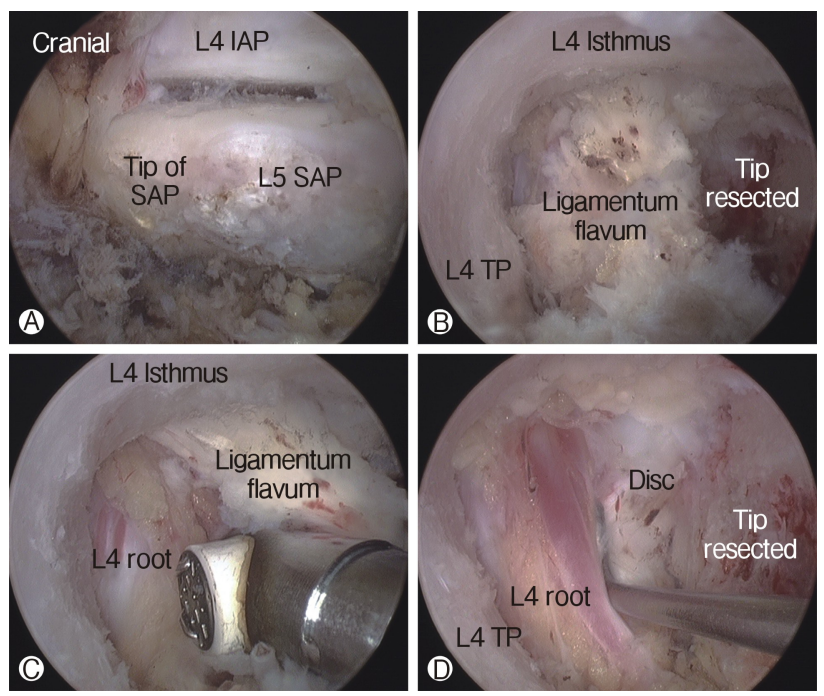

Figure 4. (A): Left-side approach endoscopic foraminal view. Exposure of the tip of superior articular process (SAP) is first step for entering the foramen. (B): After resection of the tip of SAP, the ligamentum flavum covering the exiting root was identified. (C): Under the $L F$, the exiting root was located. (D): After removal of LF, all foraminal anatomy was identified.

\section{3) Foraminal Decompression and Partial Facetectomy}

The tip of the superior articular process (SAP) of the facet joint was resected and removed using a curved chisel, pituitary
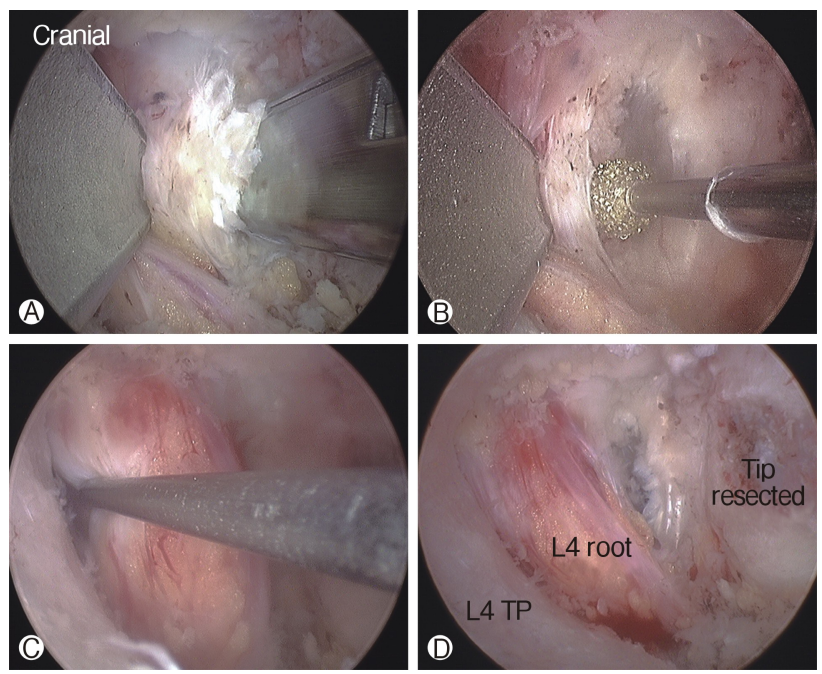

Figure 5. (A): Left-side approach endoscopic foraminal view. Discectomy using Kerrison punch. (B): Bony spur can be easily resected by endoscopic burr. (C): Identify the cranial surface of root using hook. (D): Decompression procedure around the root was done.

rongeur, and Kerrison punch. It is difficult to remove the resected tip of the SAP at once under the endoscope due to its large size; therefore, removing the tip of the SAP is a time- consuming process. After resection of the tip of the SAP, the ligamentum flavum was detached and removed from the undersurface of the isthmus of the lamina (the entrance of foramen) using a specially designed angled curette, upward-curved Kerrison punch, and upward-curved pituitary rongeur. After flavectomy, the exiting nerve was exposed and the decompression procedure around the nerve was performed using the Kerrison punch and curettes (Figure 4). An endoscopic burr was used to enlarge the roof of the foramen by thinning out the lateral edge of the isthmus, followed by the Kerrison punch. A $30^{\circ}$ endoscope was then used to visualize and perform the decompression procedure around the traversing nerve root (Figure 5).

\section{4) Final Check Point and Closure}

The end point of decompression is the free mobilization of the exiting nerve root and the dural sac containing the traversing nerve root. Additional discectomy was performed in the case of disc protrusion or extrusion contributing to the foraminal stenosis. If an annulotomy was required, it could be performed using a Penfield dissector or micro-knife. After adequate hemostasis with a radiofrequency probe, a drain was inserted through the working portal and the endoscope and instruments were extracted. The skin was repaired with a skin bond after a 1-point subcutaneous suture.

\section{5) Endoscopic Foraminal Decompression with a Contra- lateral Approach}

In case of lumbar spinal stenosis, patients usually have several types of stenosis with (1) hypertrophic ligamentum flavum and 


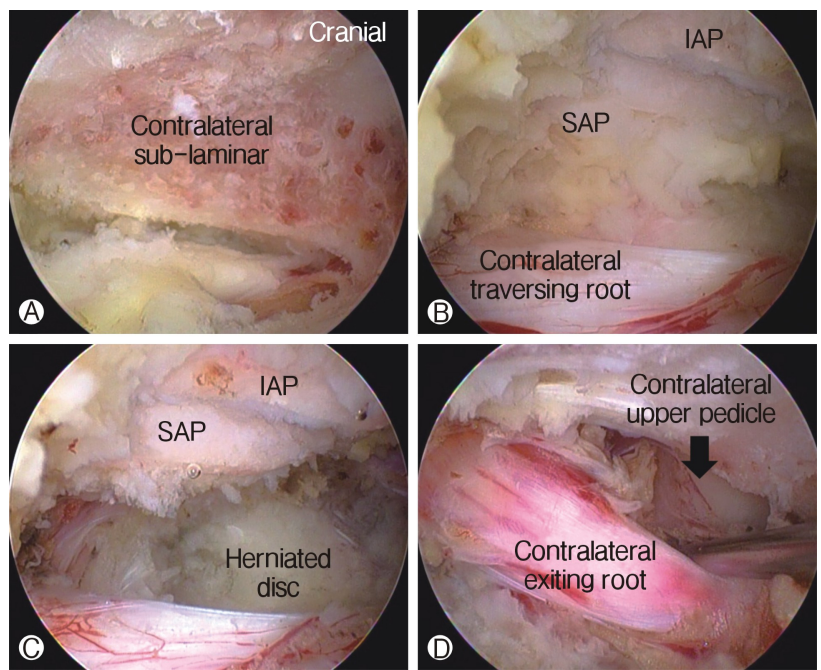

Figure 6. (A): Right-side approach and Left-side contralateral decompression. Sub-laminotomy is important to make working space. (B): After contralateral flavectomy, contralateral traversing root is easily identified. Superior articular process (SAP) and inferior articular process (IAP) were seen. (C): The herniated disc should be removed to decompress the traversing root. (D): After partial removal of the tip of superior articular process SAP, contralateral exiting root and medial wall of pedicle are identified.

disc protrusion leading to lateral recess stenosis, (2) foraminal compression with foraminal disc protrusion, syndesmophytes, and facet hypertrophy decreasing the anteroposterior diameter or overriding the superior articular facet, decreasing foraminal height with pedicle impingement, and (3) extraforaminal decompression. Sometimes, two separate approaches to decompress both lateral recess and foraminal/extraforaminal nerve root compression are required $^{31)}$. Using the advantage of more freedom to manipulate instruments under endoscopic vision, surgeons use the contralateral approach to access traversing and exiting nerve roots through a single interlaminar window. Undercutting of the contralateral hypertrophied ligamentum flavum and the SAP resection could be performed under direct endoscopic vision. Central stenosis along with foraminal or lateral recess stenosis could be decompressed effectively using a contralateral approach with minimal violation of the facet joint (Figure 6) ) $^{3}$.

By manipulating and tilting the endoscope, the undercutting of the central and contralateral lamina can be achieved, while the deep layer of the contra-lateral ligamentum flavum (LF) is prevented from expanding the dural sac. After removing the deep layer of the contra-lateral LF with rongeurs, pituitary forceps, and SAP resection with a curved chisel, the whole ventral dural sac is visualized. The lateral margin of the contralateral dura is followed by a dural retractor, and the shoulder of the contralateral traversing nerve root is visualized. This area is further decompressed using a curved Kerrison rongeur and angled curettes. The lateral margin of the dura of the traversing nerve root is visualized without constriction when it is easily retracted using a ball-tipped nerve hook. The endoscope is angled cranially to decompress the exiting nerve root on the contrala- teral side of the foraminal region. The contralateral lower lamina of the cranial vertebra is drilled off further with the burr, until the exiting nerve root at the foraminal region is visible. At this point, the foraminal area is decompressed with an angled bone curette and curved foraminotomy rongeurs until the exiting nerve root is visualized free of constrictions; it is also retractable with a ball-tipped nerve hook.

\section{DISCUSSION}

\section{Foraminal Stenosis Classification}

Lee's foraminal stenosis classification was commonly used to define the location of the offending pathology within the foramen by dividing it from medial to lateral into the entry (dural to pedicle; zone 1), middle (medial pedicle wall to center pedicle; zone 2) and exit (center pedicle to the lateral border of the facet joint; zone 3) zones ${ }^{22)}$. In the entry zone, Lee described hypertrophy of the superior articular facet as the predominant pathology. In the middle zone, foraminal stenosis was often due to an osteophytic process underneath the pars interarticularis, and in the exit zone, stenosis was typically based on facet joint subluxation and hypertrophy ${ }^{22)}$. The MRI grading of the exiting nerve root compression in the foramen also could be evaluated using the categories of Lee's classification. Grade 0 refers to normal foramen, grade 1 describes mild foraminal stenosis showing perineural fat obliteration in two opposing directions, grade 2 signifies moderate foraminal stenosis showing perineural fat obliteration in four directions, and grade 3 represent severe foraminal stenosis showing morphologic changes in the nerve $\operatorname{root}^{23)}$.

\section{Spinal Instability}

Spinal stability after conventional spinal surgery has always been an issue. Many surgeries have been performed due to spinal instability yet, there is no clear definition of spinal instability. It is most widely believed that the loss of the normal spinal pattern of motion causes pain or neurologic dysfunction ${ }^{26)}$. Other definitions of instability include increased anteroposterior translation, pathologic coupled motion, increased neutral zone, or pathologic instantaneous center of rotation, but these are findings that are also common in the normally aging spine. Thus, the exact method of determining spinal instability is unclear and it has been suggested that instability may not be very helpful in clinical practice ${ }^{21)}$. Therefore, even though prior data are questionable with the usage of the term stability, it has been believed that any significant removal of the facet joint is associated with some degree of instability of the vertebral spine ${ }^{1)}$. Important structural ligaments that can be affected with conventional facetectomy include the supraspinous, interspinous, and intertransverse ligaments along with the superficial thoracolumbar fascia and various interspinous muscles depending on the surgical approach and technique. It is unknown, whether a minimally invasive approach could allow a facetectomy to be per- 
formed with lesser instability effect like tissue-sparing conventional approach, since the trauma to the ligaments and other structural elements would be reduced ${ }^{13)}$.

Instability after resection of the SAP may be of concern. Biomechanical studies have reported that graded facetectomy greater than 50\% markedly affect the translational and rotational stabilities of the movable segment ${ }^{1,13)}$. Teo et al. ${ }^{29)}$ suggested that fixation or fusion to restore the strength and stability of the lumbar spine may be required during more than $75 \%$ of facetectomy. In a biomechanical study using finite-element models, Kiapour et al. ${ }^{18)}$ showed that $50 \%$ and $75 \%$ of facetectomy exhibited motion similar to those of the intact model at all loadings, but total facetectomy showed abnormal motion in both extension and axial rotation. With endoscopic partial facetectomy, the tip of the SAP is resected for decompression, which allows an approach to the foraminal lesion, as described above. Because the facetectomy during biportal endoscopic decompression is limited to the tip of the SAP, this technique may not be associated with postoperative instability. However, more research is needed to confirm long term results.

\section{Advantages of Biportal Endoscopic Foraminal Decompression Over Full Endoscopic Procedure}

The primary advantages of endoscopy-assisted spinal surgery have been reported in several papers ${ }^{3,8,20)}$. Recently introduced bi-portal endoscopic spinal surgery has advantages over the full endoscopic spinal surgery in the far lateral approach. First, the disadvantage of full endoscopic decompression is the risk of a possible injury to the exiting nerve root during the procedure, if the foraminal space is severely narrow and the working cannula is not positioned appropriately. The floating-type biportal endoscopic technique can be used with various surgical instruments that cannot be used in full endoscopic surgery, unlike in the docking-type technique, such as conventional Kerrison rongeurs, pituitary forceps, various burrs, osteotomes or chisels, regardless of size. It also has the advantage of covering a wide working space because it does not stay in one compartment with docking.

Secondly, far lateral stenosis caused by L5-S1 level of the hypertrophied sacral ala is limited by the full endoscopic technique, whereas the biportal endoscopic technique is able to solve the body decompression by instruments such as the osteotome and the endoscopic drill via another portal. Third, as a condition for successful decompression, the SAP in the foramen must be completely removed until the ligamentum flavum is exposed, and the exiting root must be fully decompressed from the entrance of the foramen to the extraforaminal area. However, in the case of open microscopic decompression or full endoscopic decompression, access to the inside of the L5-S1 foramen is not easy due to the iliac crest. With biportal endoscopic spine surgery, multiple sites can be accessed with more freedom of approaching angle through triangulation and portal switching. When using a $30^{\circ}$ endoscopy, various views can be obtained through rotation.

\section{Outcome Factors with Foraminal Decompression}

Open decompression surgery using the Wilte approach is a conventional decompression surgery for extraforaminal or foraminal stenosis. Open microscopic foraminotomy reports a success rate of $72-83 \%$, though it has a lower success rate compared to other spinal surgeries ${ }^{7}$. In addition, excessive dissection of the paraspinal muscle may cause back pain or muscle atrophy. Recent advances in optic and endoscopic devices have allowed better vision and more precise operation; good results of decompression surgery in the foramen or extraforaminal stenosis using endoscope have been reported ${ }^{3,8,20)}$.

Several authors have serendipitously found that actual symptoms do not always correspond to the imaging degree of foraminal stenosis ${ }^{12)}$. Although CT and MRI imaging measurements are useful guidelines in the evaluation of lumbar stenosis conditions, the actual symptoms do not always correspond to traditional imaging studies. Also, it is not easy to objectively evaluate clinical outcome through corrections of imaging after decompression. However, we would like to consider the poor outcome factors mentioned by several authors and discuss the appropriate indications for endoscopic foraminal decompression.

\section{1) Lumbar Lordosis}

It is well known that sagittal spinal balance is significantly associated with the postoperative quality of life of patients with spinal deformities ${ }^{10)}$. Lumbar lordosis is an indicator that reflects the local alignment of the lumbar spine. Some recent studies have investigated the relationship between lumbar lordosis and the surgical outcome of spinal stenosis ${ }^{5,11)}$. Chang reported that preoperative lumbar lordosis significantly influenced the surgical outcome of lumbar spinal stenosis ${ }^{5}$. Haimoto et al. ${ }^{11)}$ also reported that reduction of lumbar lordosis is predictive of restenosis after microscopic foraminal decompression surgery. Their study demonstrated that the clinical outcomes were poor for patients with little improvement of lumbar lordosis postoperatively.

\section{2) Degenerative Lumbar Scoliosis}

Yamada et al. ${ }^{32)}$ suggested degenerative lumbar scoliosis as a risk factor for poor outcomes after microsurgical foraminotomy of the lumbar spine. They reported a high recurrence rate and poor clinical improvement in patients with degenerative lumbar scoliosis. Yoshimoto et al. ${ }^{33)}$ reported that degenerative lumbar scoliosis progression caused by degenerative change, such as lateral disc space wedging, may directly lead to the recurrence of foraminal up-down stenosis. There are a few reports about risk factors for curve progression after decompression surgery in the literature. Hosogane et al. ${ }^{14)}$ reported that bony spur formation on the concave side of the curve, which might stabilize the spine, and may reduce curve progression after decompression. It is necessary to determine whether it would be beneficial to remove the SAP sufficiently and achieve sufficient decompression or whether this makes degenerative lumbar scoliosis worse. Further studies are needed about lumbar curve progre- 
ssion after endoscopic foraminal decompression.

\section{3) Posterior Intervertebral Disc Height and Operation Level}

The heights of the posterior intervertebral disc could be one of the factors that affect clinical outcomes because disc space narrowing is likely to cause root compression. Lewandrowski et $\mathrm{al}^{25}{ }^{25}$ reported that a vacuum and collapsed disc ( $<3 \mathrm{~mm}$ posterior disc height) is associated with less favorable outcomes after endoscopic foraminal decompression. However, in many cases, root compression was not severe in the foramen, although disc space narrowing was severe. Youn et al. ${ }^{35)}$ reported no statistically significant association between disc height and the clinical outcomes. Some studies have reported relatively poor surgical outcomes after facet-preserving decompression, more often at the L5-S1 level than at the other surgical levels ${ }^{30,31)}$. Bae et al. ${ }^{4}$ reported a poor outcome rate of $22.2 \%$ for patients who had undergone foraminotomy with a microscope at the L5-S1 level. Also, revision surgery was more common at the L5-S1 level than at other operative levels ${ }^{4}$.

\section{How to Avoid Complications}

There must be several technical pitfalls and complications in the learning curve of biportal endoscopic foraminal decompression. Muscle edema, hydro-peritoneum, incomplete decompression, blurred surgical view, and injury of the radicular artery due to unfamiliar or misunderstanding of the anatomic structure were present. Muscle edema occurred due to a poor saline output; inserting a cannula through the portal could be helpful. If orientation were lost without touching the transverse process, the psoas muscle layer could become penetrated, and infused saline could make a hydro-peritoneum. If it is difficult to determine the exact location of the structure, the positioning of the endoscope should be rechecked using a fluoroscope.

Controlling small bleeds from the vessels and radicular artery was very important to make a clear surgical field and not to injure the root. A blurred surgical field due to small bleedings interrupted the procedure frequently. Small vascular bleeding could be controlled using $1.5 \mathrm{~mm}$ radiofrequency coagulation under the very lowest setting of energy. The hypertrophied tip of the SAP should be removed sufficiently to decompress the root and prevent dynamic stenosis at the foramen ${ }^{8)}$. Instability after resection of the SAP may be of concern, and the tip of the SAP should be gradually removed until there is enough foraminal decompression. It is difficult to remove the resected tip of the SAP all at once under the endoscope due to its large size; therefore, more time is required to complete this process.

Careful analysis of CT and MRI images is vital to planning the amount of foraminal decompression with consideration of the anteroposterior and superior-inferior diameter of the foramen. Such planning avoids unnecessary endoscopic foraminal exploration. When intraforaminal lesions are treated, it is important to use a $30^{\circ}$ endoscope with a specially designed angled curette, an upward-curved Kerrison punch, and an upwardcurved pituitary rongeur. Clinical findings with concordant imag- ing are key in patient selection. Preoperative clinical and radiological assessments for gross anteroposterior and lateral instability are essential. We need further research to predict poor progression factors after endoscopic foraminal decompression.

It is not clear yet which indication is best for biportal endoscopic foraminal decompression. The best indications and contraindications to the endoscopic foraminal decompression still depends on the surgeon's skill level and evolving experience. Also, several papers suggested less durable outcomes in patients with complex foraminal stenotic lesion and combined severe central stenosis. Stratifying patients based on the underlying compressive pathology and appropriate skill level of the endoscopic spine surgeon attempting the decompression may facilitate achieving the goals of the endoscopic surgery ${ }^{24)}$.

\section{CONCLUSION}

Through the studies about endoscopic partial facetectomy for foraminal stenosis, the results revealed that endoscopic facectectomy seems not to alter spinal stability when compared with conventional surgeries. We hypothesize that this is due to the reduced tissue destruction similar to that seen with tissue sparing conventional facetectomy. Thus, the ligament structure of the spine is held mainly intact which has been shown to be important for the structural integrity of the spine. Biportal endoscopic spinal surgery is supposed to be an alternative treatment for foraminal decompression in various foraminal stenosis conditions while preserving motion and stability and decreasing the need for fusion surgery to relieve various lumbar degenerative diseases.

\section{CONFLICT OF INTEREST}

No potential conflict of interest relevant to this article.

\section{REFERENCES}

1. Abumi K, Panjabi MM, Kramer KM, Duranceau J, Oxland T, Crisco JJ: Biomechanical evaluation of lumbar spinal stability after graded facetectomies. Spine (Phila Pa 1976) 15:1142-1147, 1990

2. Ahn Y, Oh HK, Kim H, Lee SH, Lee HN: Percutaneous endoscopic lumbar foraminotomy: An advanced surgical technique and clinical outcomes. Neurosurgery 75:124-133; discussion 132123, 2014

3. Akbary K, Kim JS, Park CW, Jun SG, Hwang JH: Biportal Endoscopic Decompression of Exiting and Traversing Nerve Roots Through a Single Interlaminar Window Using a Contralateral Approach: Technical Feasibilities and Morphometric Changes of the Lumbar Canal and Foramen. World Neurosurg 117:153161, 2018

4. Bae JS, Kang KH, Park JH, Lim JH, Jang IT: Postoperative Clinical Outcome and Risk Factors for Poor Outcome of Foraminal and Extraforaminal Lumbar Disc Herniation. J Korean Neurosurg Soc 59:143-148, 2016 
5. Chang HS: Influence of Lumbar Lordosis on the Outcome of Decompression Surgery for Lumbar Canal Stenosis. World Neurosurgery 109:e684-e690, 2018

6. Chang SB, Lee SH, Ahn Y, Kim JM: Risk factor for unsatisfactory outcome after lumbar foraminal and far lateral microdecompression. Spine (Phila Pa 1976) 31:1163-1167, 2006

7. Cho S-I, Chough C-K, Choi S-C, Chon JY: Microsurgical Foraminotomy via Wiltse Paraspinal Approach for Foraminal or Extraforaminal Stenosis at L5-S1 Level: Risk Factor Analysis for Poor Outcome. Journal of Korean Neurosurgical Society 59: 610-614, 2016

8. Choi D-J, Kim J-E, Jung J-T, Kim Y-S, Jang H-J, Yoo B, et al.: Biportal Endoscopic Spine Surgery for Various Foraminal Lesions at the Lumbosacral Lesion. Asian Spine Journal 12:569-573, 2018

9. Epstein NE: Foraminal and far lateral lumbar disc herniations: Surgical alternatives and outcome measures. Spinal Cord 40:491500, 2002

10. Glassman SD, Bridwell K, Dimar JR, Horton W, Berven S, Schwab F: The impact of positive sagittal balance in adult spinal deformity. Spine (Phila Pa 1976) 30:2024-2029, 2005

11. Haimoto S, Nishimura Y, Hara M, Nakajima Y, Yamamoto Y, Ginsberg HJ, et al.: Clinical and Radiological Outcomes of Microscopic Lumbar Foraminal Decompression: A Pilot Analysis of Possible Risk Factors for Restenosis. Neurologia medico-chirurgica 58:49-58, 2018

12. Hasegawa T, An HS, Haughton VM, Nowicki BH: Lumbar fora minal stenosis: Critical heights of the intervertebral discs and foramina. A cryomicrotome study in cadavera. J Bone Joint Surg Am 77:32-38, 1995

13. Haufe SM, Mork AR: Effects of unilateral endoscopic facetectomy on spinal stability. J Spinal Disord Tech 20:146-148, 2007

14. Hosogane N, Watanabe K, Kono H, Saito M, Toyama Y, Matsumoto M: Curve progression after decompression surgery in patients with mild degenerative scoliosis. J Neurosurg Spine 18: 321-326, 2013

15. Jenis LG, An HS: Spine update. Lumbar foraminal stenosis. Spine (Phila Pa 1976) 25:389-394, 2000

16. Kambin P, Sampson S: Posterolateral percutaneous suction-excision of herniated lumbar intervertebral discs. Report of interim results. Clin Orthop Relat Res: 37-43, 1986

17. Kang K, Rodriguez-Olaverri JC, Schwab F, Hashem J, Razi A, Farcy JP: Partial facetectomy for lumbar foraminal stenosis. Adv Orthop 2014:534658, 2014

18. Kiapour A, Ambati DV, Hoy RW, Goel V: Effect of Graded Facetectomy on Biomechanics of Dynesys Dynamic Stabilization System. Spine 37:E58Q E589, 2012

19. Kim HS, Adsul N, Kapoor A, Choi SH, Kim JH, Kim KJ, et al.: A Mobile Outside-in Technique of Transforaminal Lumbar Endoscopy for Lumbar Disc Herniations. J Vis Exp, 2018

20. Kim JE, Choi DJ: Bi-portal Arthroscopic Spinal Surgery (BASS) with $30^{\circ}$ arthroscopy for far lateral approach of L5-S1 - Technical note. J Orthop 15:354-358, 2018

21. Krismer M, Haid C, Ogon M, Behensky H, Wimmer C: [Biomechanics of lumbar instability]. Orthopade 26:516-520, 1997

22. Lee CK, Rauschning W, Glenn W: Lateral lumbar spinal canal stenosis: Classification, pathologic anatomy and surgical decompression. Spine (Phila Pa 1976) 13:313-320, 1988

23. Lee S, Lee JW, Yeom JS, Kim KJ, Kim HJ, Chung SK, et al.: A practical MRI grading system for lumbar foraminal stenosis. AJR Am J Roentgenol 194:1095-1098, 2010

24. Lewandrowski K-U, Dowling Á, de Carvalho PST, Calderaro AL, dos Santos TS, de Lima e Silva MS, et al.: Indication and Contraindication of Endoscopic Transforaminal Lumbar Decompression. World Neurosurgery 145:631-642, 2021

25. Lewandrowski KU, León JFR, Yeung A: Use of "Inside-Out" Technique for Direct Visualization of a Vacuum Vertically Unstable Intervertebral Disc During Routine Lumbar Endoscopic Transforaminal Decompression-A Correlative Study of Clinical Outcomes and the Prognostic Value of Lumbar Radiographs. Int J Spine Surg 13:399-414, 2019

26. Panjabi MM: Clinical spinal instability and low back pain. Journal of Electromyography and Kinesiology 13:371-379, 2003

27. Rauschning W: Pathoanatomy of lumbar disc degeneration and stenosis. Acta Orthopaedica Scandinavica 64:3-12, 1993

28. Reulen HJ, Pfaundler S, Ebeling U: The lateral microsurgical approach to the "extracanalicular" lumbar disc herniation. I: A technical note. Acta Neurochir (Wien) 84:64-67, 1987

29. Teo EC, Lee KK, Qiu TX, Ng HW, Yang K: The biomechanics of lumbar graded facetectomy under anterior-shear load. IEEE Trans Biomed Eng 51:443-449, 2004

30. Wiltse LL, Spencer CW: New uses and refinements of the paraspinal approach to the lumbar spine. Spine (Phila Pa 1976) 13: 696-706, 1988

31. Wu PH, Kim HS, Jang I-T: How I do it? Uniportal full endoscopic contralateral approach for lumbar foraminal stenosis with double crush syndrome. Acta Neurochirurgica 162:305-310, 2020

32. Yamada K, Matsuda H, Nabeta M, Habunaga H, Suzuki A, Nakamura H: Clinical outcomes of microscopic decompression for degenerative lumbar foraminal stenosis: A comparison between patients with and without degenerative lumbar scoliosis. Eur Spine J 20:947-953, 2011

33. Yoshimoto M, Iesato N, Terashima Y, Tanimoto K, Oshigiri T, Emori M, et al.: Mid-term Clinical Results of Microendoscopic Decompression for Lumbar Foraminal Stenosis. Spine Surg Relat Res 3:229-235, 2019

34. Youn MS, Shin JK, Goh TS, Lee JS: Clinical and radiological outcomes of endoscopic partial facetectomy for degenerative lumbar foraminal stenosis. Acta Neurochir (Wien) 159:1129-1135, 2017

35. Youn MS, Shin JK, Goh TS, Lee JS: Predictors of Clinical Outcome After Endoscopic Partial Facetectomy for Degenerative Lumbar Foraminal Stenosis. World Neurosurg 126:e1482-e1488, 2019 